\title{
Differential Space Time Shift Keying for Dispersive Multi-user Scenarios using OFDM
}

\author{
S.Ruth Karunya \\ Department of Information \& Communication \\ Engineering, \\ Anna University Chennai, Regional Center \\ Madurai \\ Madurai, India.
}

\author{
S.Veluchamy \\ Department of Information \& Communication \\ Engineering, \\ Anna University Chennai, Regional Center \\ Madurai \\ Madurai, India.
}

\begin{abstract}
Spatial modulation (SM) is a recently developed transmission technique that uses multiple antennas. The concept of SM is extended to both the space and time dimensions to provide a general shift-keying framework. A new modulation scheme called Differential STSK (DSTSK) scheme, assisted by the Cayley unitary transform is proposed to OFDM aided Multiple Input Multiple Output (MIMO) Communication Systems. The main advantage of this modulation technique is that it does not require any Channel State Information (CSI) at the receiver. More specifically, by employing the Cayley transform in the proposed DSTSK scheme we arrive at a linearized equivalent system model. Cayley codes encode the transmitted data differentially using unitary matrices at the transmitter, and hence the transmitted data can be decoded differentially at the receiver without knowing the channel coefficients. Since channel knowledge is not required at the receiver, differential schemes are ideal for use on wireless links where channel tracking is undesirable or infeasible, due to rapid changes in the channel characteristics or limited system resources. Further the proposed scheme enables us to achieve a flexible diversity and multiplexing gain tradeoff.
\end{abstract}

Keywords -,Dispersive Channel, Differential Space Time shift Keying (DSTSK), Cayley Unitary Transform, Hermitian Matrix, Channel State Information(CSI)

\section{INTRODUCTION}

Reliable mobile wireless transmission of video, data, and speech at high rates to many users is an important part of future telecommunications systems. Over the past fifteen years, the bit rates achieved in cellular and local area wireless communications systems have increased steadily. The highest bit rates in commercially deployed wireless systems can be achieved by means of Orthogonal Frequency Division Multiplexing (OFDM) High data rate and high spectral efficiency are the key elements in future wireless communication systems. Space-time coding (STC), multiple antennas and multiple-input-multiple output (MIMO) systems, multicarrier modulation, and ultra wideband radio are examples of next generation wireless communication technologies. Among the set of existing technologies, MIMO orthogonal frequency division multiplexing (MIMO-OFDM) is a very promising technology for future wireless systems. MIMO refers to multiple Antenna Elements (AEs) employed at a transmitter and/or a receiver. A MIMO system boosts spectral efficiency by using multiple antennas to simultaneously transmit data to the receiver.

MIMO technology has attracted attention in wireless communications, because it offers significant increases in data throughput and link range without additional bandwidth or increased transmit power. MIMO can be sub-divided into three main categories, precoding, spatial multiplexing or SM, and diversity coding.

Multi-user OFDM is used to mitigate intersymbol interference and enhance system capacity in wideband transmission OFDM allows for a high spectral efficiency as the carrier power and modulation scheme can be individually controlled for each carrier. OFDM can be applied in a multiuser application producing a highly flexible, efficient communication system.

Most OFDM systems use a fixed modulation scheme over all carriers. However each carrier has a different modulation scheme depending on the channel conditions. Most of the modulation schemes provide a trade off between the spectral efficiency and bit error rate. The spectral efficiency can be maximized by choosing the highest modulation scheme that will give an acceptable bit error rate. Hence carrier modulation must be designed to provide an acceptable Bit error rate even under the worst channel conditions. Systems using BPSK or QPSK result in poor spectral efficiency and provide excess link margin most of the time.

Recently, the sophisticated concept of Spatial Modulation (SM) [7]-[9] and Space-Shift Keying (SSK) [10] was invented for Multiple-Input Multiple-Output (MIMO) communication systems. The key idea is the activation of one of a total of M Antenna Elements (AEs) at each symbol duration, leading to an additional means of conveying source information, while removing the effects of ICI. Space-Time Shift Keying (STSK) modulation, which constitutes a generalized shift-keying architecture thereby implementing the modulation scheme in both space as well as time dimensions. In this paper, we introduce an improved STSK structure, called Differential Space Time Shift Keying (DSTSK) which enables us to dispense with any symbol-level timesynchronization between the transmit AEs.

Although DSTSK-based systems have an excellent performance in narrowband channels, their performance in dispersive wireless channels may erode. To mitigate 
the performance degradation imposed by dispersive channels, we introduce Orthogonal Frequency Division Multiplexing(OFDM) concept along with Differential Space Time Shift Keying (DSTSK).

The rest of this paper is organized as follows. In Section II the proposed Differential Space Time Shift Keying (DSTSK) is explained. In Section III, we present a brief overview of our proposed OFDM aided DSTSK system model. Then, the performance of the scheme, particularly of an EXIT chart aided near-capacity design is investigated in Section IV. Finally, we conclude in Section V.

\section{RELATED WORK}

Multiple antenna aided wireless communication systems have attracted substantial attention due to their ability of achieving reliable high-rate transmission The Vertical Bell Laboratories Layered Space-Time (VBLAST) [11] scheme is capable of attaining a high multiplexing gain at the cost of a substantial decoding complexity imposed by mitigating the effects of InterChannel Interference (ICI). By contrast, Space-Time Block Codes (STBCs) [1], [3] were developed to achieve the maximum attainable diversity order, although the maximum bandwidth efficiency of the full-rate orthogonal STBCs is limited to one bit per symbol duration. Linear Dispersion Codes (LDCs), which subsumes both the V-BLAST and Alamouti's STBC scheme, was proposed in [15]. Additionally, in [9] the differential-encoding assisted counterpart of LDCs was introduced in order to enable non-coherent detection at the receiver in the absence of CSI, which was referred to as Differential LDC (DLDC). Recently, the sophisticated concept of Spatial Modulation (SM) [5],[8] and SpaceShift Keying (SSK) [7] was invented for Multiple-Input Multiple-Output (MIMO) communication systems. The key idea is the activation of one of a total of $M$ Antenna Elements (AEs) at each symbol duration, leading to an additional means of conveying source information, while removing the effects of ICI. SM/SSK in [7] has the ability to outperform other MIMO arrangements, such as V-BLAST and Alamouti's STBC schemes.

\section{DIFFERENTIAL SPACE TIME SHIFT KEYING MODULATION}

The STSK scheme and the conventional SM/SSK scheme are both based on the prior knowledge of CSI and hence the performance degradation imposed by CSI estimation errors is unavoidable. To avoid this limitation, we proposed the corresponding DSTSK scheme as the extension of STSK scheme with the aid of the Cayley unitary transform proposed in [2] which eliminates the need for Channel State Information (CSI) at the receiver.

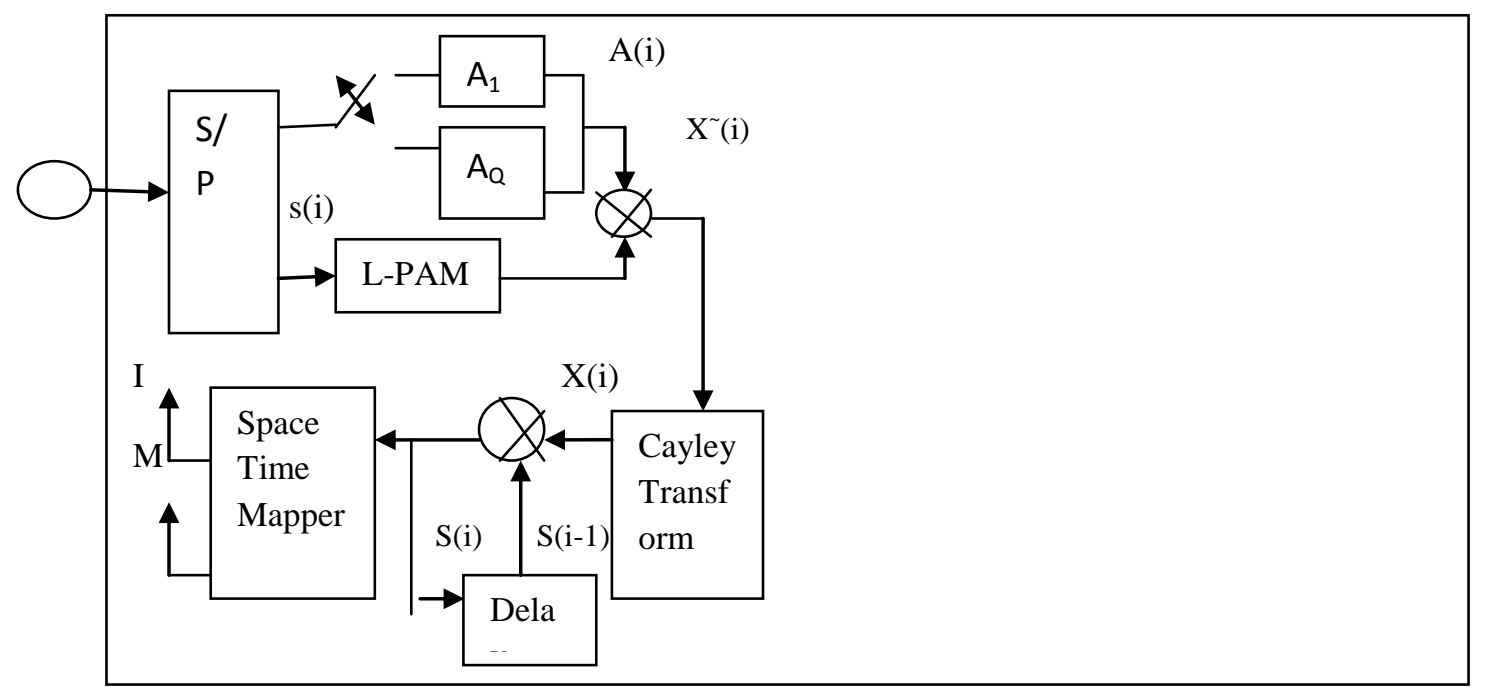

Figure 1: Transmitter of DSTSK scheme

The transmitter structure of our DSTSK scheme is shown in figure 1 .Hermitian matrices $\mathrm{A}_{\mathrm{q}}(\mathrm{q}=1,2, \ldots \mathrm{Q})$ are preallocated as the dispersion matrices prior to transmissions and L-level Pulse Amplitude Modulation (PAM) is employed. each space-time block contains $\log _{2}(\mathrm{Q} . \mathrm{L})$ source bits, where $\log _{2} \mathrm{Q}$ bits are mapped to $\mathrm{A}(\mathrm{i})$ using the previously outlined process of dispersion matrix activation, while $\log _{2} \mathrm{~L}$ bits are mapped to the L- PAM symbols $\mathrm{s}(\mathrm{i})$. the Hermitian matrix $\mathrm{X}^{\sim}(\mathrm{i}) \mathrm{C}^{\mathrm{M} * \mathrm{~T}}$ is calculated as follows

$$
\mathrm{X}^{\sim}(\mathrm{i})=\mathrm{s}(\mathrm{i}) \cdot \mathrm{A}(\mathrm{i})
$$

where $\mathrm{M}=\mathrm{T}$. According to the Cayley unitary transform technique discussed in [6], the Hermitian Matrix $X^{\sim}(i)$ is transformed to unitary matrix X(i) as follows.

$$
X(i)=\left[I-j X^{\sim}(i)\right]\left[I+j X^{\sim}(i)\right]^{-1}
$$

The above equation is referred to as Cayley Unitary Transform. It uniquely connects the unitary matrix $X(i)$ with the hermitian matrix $X^{\sim}(i)$ and thereby enabling differential encoding.Inorder to ensure that the Hermitian matrix $\mathrm{X}^{\sim}(\mathrm{i})$, the modulated symbol S(i) must be a real valued signal. That is it must follow either QAM or PAM modulation. We have employed Pam modulation in this paper. 

follows

The Space time matrix is differentially encoded as

$$
\mathrm{S}(\mathrm{i})=\mathrm{S}(\mathrm{i}-1) \cdot \mathrm{X}(\mathrm{i})
$$

where the symbols in the mth row of S(i) are transmitted from the mth transmit $\mathrm{AE}$ over $\mathrm{T}$ symbol durations.

In general the received signal can be described as

$$
Y(i)=H(i) S(i)+V(i)
$$

where $\mathrm{Y}(\mathrm{i}) \mathrm{C}^{\mathrm{M} * \mathrm{~T}}$ represents the received signals and $\mathrm{S}(\mathrm{i})$ $\in \mathrm{C}^{\mathrm{M}^{* \mathrm{~T}}}$ denotes the space-time signals and the mth row's elements are transmitted from the mth antenna, while $i$ indicates the DSTSK block index. Furthermore,H(i) $\epsilon$ $\mathrm{C}^{\mathrm{N}^{* \mathrm{M}}}$ and $\mathrm{V}(\mathrm{i}) \in \mathrm{C}^{\mathrm{N}^{* \mathrm{~T}}}$ denote the channel and noise components, Assuming that the fading channel envelope remains constant over the two DSTSK block durations $2 T$, the corresponding received signal block $\boldsymbol{Y}(i)$ of Eq. (1) is modified to

$$
\mathrm{Y}(\mathrm{i})=\mathrm{Y}(\mathrm{i}-1) \mathrm{X}(\mathrm{i})+\mathrm{V}(\mathrm{i})-\mathrm{V}(\mathrm{i}-1) \mathrm{X}(\mathrm{i})
$$

which does not include any channel components. Instead of directly applying optimum ML detection to the received signal of Eq. (17), we introduce the linearization technique of [6] upon multiplying both sides of Eq. (2.5) by $[\mathrm{I}+\mathrm{jX}(\mathrm{i})]$, we arrive at

$$
Y^{\wedge}(i)=H^{\wedge}(i) \cdot X^{\sim}(i)+V^{\wedge}(i)
$$

Where $\mathrm{Y}^{\wedge}(i)$ and $\mathrm{H}^{\wedge}(i)$ represent the equivlent received signals and the equivalent channel matrix, while the equivalent noise matrix ${ }^{\wedge}(i)$ has independent columns with a covariance of

$$
\mathrm{N}_{0}^{\sim}=\mathrm{N}_{0}\left(1+\mathrm{X}^{\sim 2}\right)
$$

\section{PROPOSED SYSTEM MODEL}

In this paper,we consider an OFDM aided DSTSK system with $M$ transmit and $N$ receive Antenna elements(AEs). The transmitter and receiver structure of the proposed system model is explained as follows.

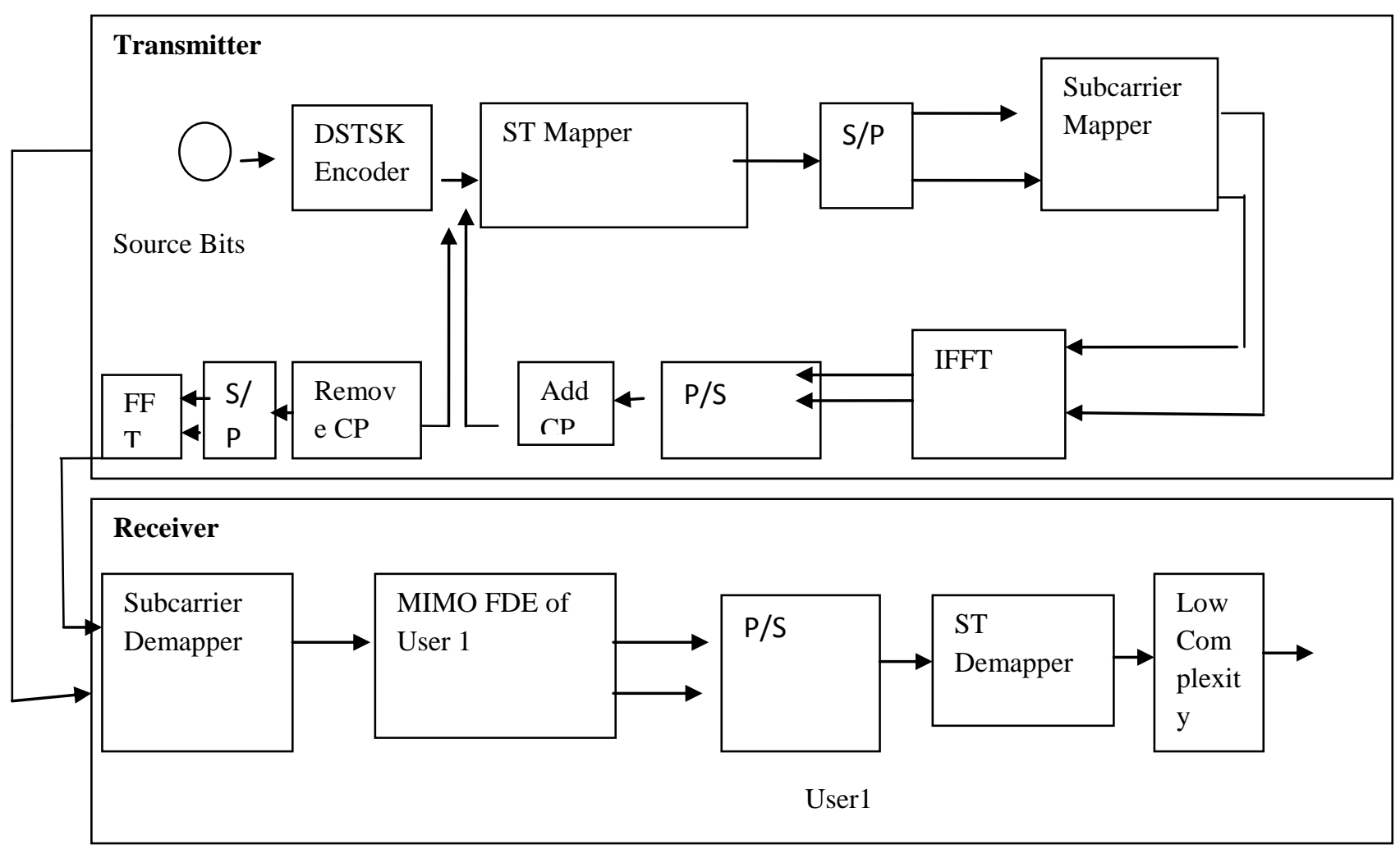

Figure 2: Block Diagram of OFDM aided DSTSK scheme

\subsection{Transmitter}

The transceiver architecture of our OFDM aided DSTSK system is shown in Fig. 2. The signals are first mapped by the space-time (ST) Mapper of the DSTSK block. DSTSK encoder in Fig. 2 maps the source information of one of the U users to ST blocks $x^{u}\left[n_{d}\right] \epsilon$ $\mathrm{C}^{\mathrm{M} * \mathrm{~T}}, \mathrm{n}_{\mathrm{d}}=0,1,2 \ldots . .\left(\mathrm{N}_{\mathrm{d}}-1\right)$

$$
x^{u}\left[n_{d}\right]=s^{u}\left[n_{d}\right] \cdot A^{u}\left[n_{d}\right]
$$

where $s^{\mathrm{u}}\left[\mathrm{n}_{\mathrm{d}}\right]$ and $\mathrm{A}^{\mathrm{u}}\left[\mathrm{n}_{\mathrm{d}}\right]$ represent the $u$ th user's L-ary phase-shift keying (PSK)/quadrature amplitude modulation (QAM) symbol and activated dispersion matrix (DM), respectively, from a set of $\mathrm{Q}$ such matrices 
Aq $(q=1,2 \ldots, Q)$, which are pre assigned in advance of transmissions. Thus, a block of $\log _{2}$ (L.Q) number of bits are transmitted by the ST mapper in Fig. 2 per symbol interval forms an STSK ST block.

After generating the ST blocks $\mathrm{x}^{\mathrm{u}}\left[\mathrm{n}_{\mathrm{d}}\right]$ for a particular user $u$, we employ frame-based transmission. In particular, $N c$ subcarriers are used for transmitting a frame, each frame consisting of Nd DSTSK blocks.

\subsection{Receiver}

After removing the cyclic prefix, the $\mathrm{N}_{\mathrm{c}}$ point IFFT is computed as follows

$$
Y=\sum_{u=0}^{U-1} \boldsymbol{H}^{\sim u} \boldsymbol{X}^{\sim u}+V
$$

where each $(n, m)$ th component of $\mathrm{H}^{\sim u}$ is formulated as

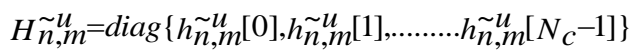

under the idealized assumption of perfect synchronization, perfect orthogonality of the users using different subcarriers, our proposed scheme becomes free from multi user interference.

Following the equalization of the receiver, the decision variable $\mathrm{zu}$ for the proposed scheme can readily be written as

$$
\begin{gathered}
z^{u^{\prime}}\left[\boldsymbol{n}_{d}\right]=x^{\sim u^{\prime}}\left[\boldsymbol{n}_{d}\right]+\boldsymbol{v}^{\sim u^{\prime}}\left[\boldsymbol{n}_{d}\right] \\
\text { Where } \\
x^{\sim u^{\prime}}\left[\boldsymbol{n}_{d}\right] \in \boldsymbol{C}^{M^{*} T} \\
\boldsymbol{v}^{\sim u^{\prime}}\left[\boldsymbol{n}_{d}\right] \in \boldsymbol{C}^{M^{* T}}
\end{gathered}
$$

single-stream-based ML detection [1] to detect the indices $q$ and $l c$ of the DM activated and the constellation symbol used, respectively. The estimates $\left(q^{\wedge}, l_{c}^{\wedge}\right)$ can be determined from the following equation

$$
\left(\hat{q}, \boldsymbol{l}_{c}^{\wedge}\right)=\underset{q, l_{c}}{\arg \min }\left\|\bar{z}^{u^{\prime}}\left[\boldsymbol{n}_{d}\right]-\chi k_{q, l_{c}}^{u^{\prime}}\right\|^{2}
$$

Finally the OFDM aided STSK signal can be detected from the above equation.

\section{SIMULATION RESULTS}

The following are the simulation results of a $2 * 2 \mathrm{MIMO}$ OFDM system.

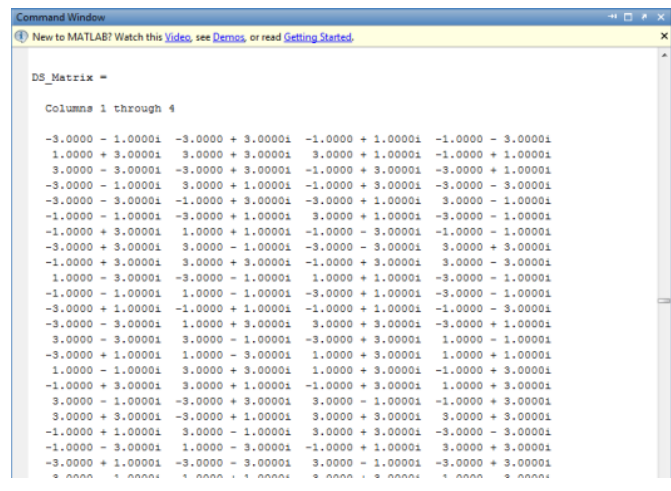

Figure 3: Generation of Dispersion Matrix

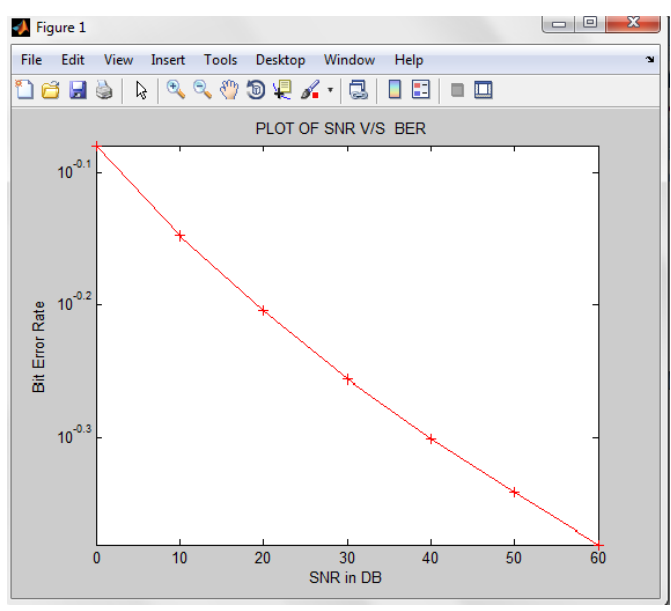

Figure 4: Plot of Signal to Noise Ratio Vs Bit Error rate (BER)

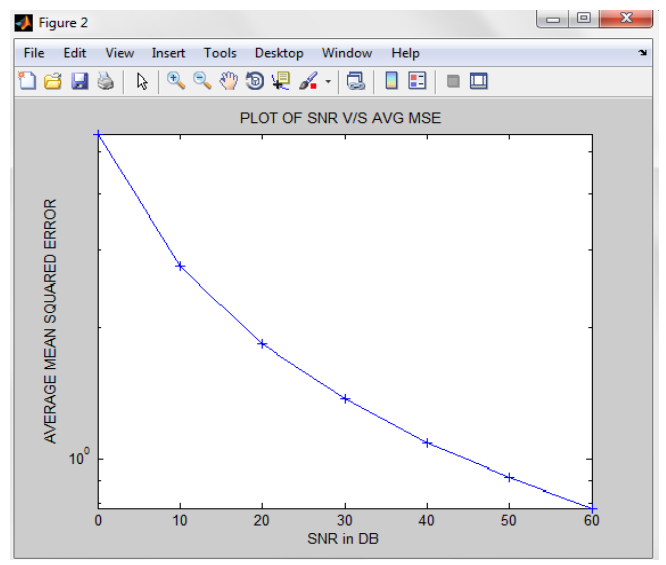

Figure 5: Plot of Signal to Noise Ratio (SNR) Vs Average Minimum Mean Square Error (MMSE) 


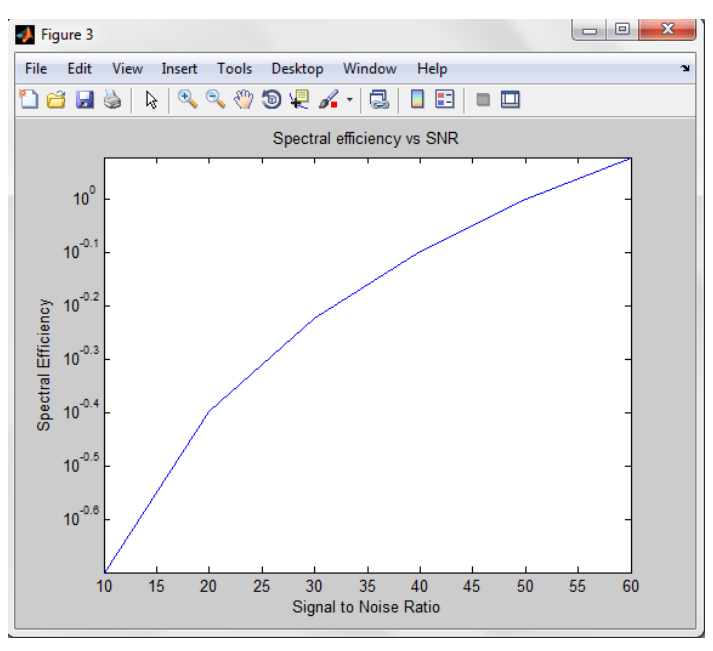

Figure 6: Plot of Spectral Efficiency Vs Signal to Noise Ratio (SNR)

Table 1: Main Simulation Parameters of Proposed Scheme

\begin{tabular}{|l|l|}
\hline \multicolumn{1}{|c|}{ Simulation Parameter } & \multicolumn{1}{c|}{ Value } \\
\hline No of Subcarriers & 128 \\
\hline Size of FFT & 2048 \\
\hline $\begin{array}{l}\text { No of Transmit antenna elements, } \\
\text { M }\end{array}$ & 2 \\
\hline No of receive antenna elements, N & 2 \\
\hline No of Transmit time slots, T & 2 \\
\hline No of Dispersion Matrices, Q & 2 \\
\hline DSTSK Specification & $(2,2,2, \mathrm{Q})$ \\
\hline Modulation Order & 2 \\
\hline
\end{tabular}

The above table gives the values of the simulation parameters of the proposed scheme.

\section{ACKNOWLEDGEMENT}

The authors would like to thank the anonymous reviewers for providing constructive comments and suggestions that have contributed to the improvement in the quality and presentation of this paper.

\section{CONCLUSION AND FUTURE WORK}

In this paper, an OFDM aided DSTSK scheme has been proposed, which overcomes the need for estimating Channel State Information(CSI) at the receiver .It also results in a sharp decrease of the Bit Error Rate (BER) curve. In future, the effects of spatial correlation between different transmit elements has to be investigated and to produce a very low Bit Error Rate (BER) curve.

\section{REFERENCES}

[1] Alamouti . S (1998), "A simple transmit diversity technique for wireless communications," IEEE J. Sel. Areas Communication., vol. 16, no. 8, pp. 1451-1458.

[2] Babak Hassibi and Bertrand Hochwald .M (2002), "Cayley differential unitary space-time codes," IEEE Trans. Inf. Theory, vol. 48, no. 6, pp. 14851503.

[3] Babak Hassibi and Bertrand Hochwald .M (2002), "High-Rate Codes That Are Linear in Space and Time" IEEE Transactions On Information Theory, Vol. 48, No. 7.

[4] Heath R.W, Jr. and Paulraj .A (2002), "Linear dispersion codes for MIMO systems based on frame theory," IEEE Trans. Signal Process., vol. 50, no. 10, pp. 2429-2441.

[5] Jeganathan .J, Ghrayeb .A, and Szczecinski .L (2008), "Spatial modulation: optimal detection and performance analysis," IEEE Commun. Letters., vol. 12 , no. 8 , pp. 545-547..

[6] Jeganathan .J, Ghrayeb .A(2008), and Szczecinski .L, "Generalized space shift keying modulation for MIMO channels," in Proc. IEEE $19^{\text {th }}$ International Symp. Personal, Indoor Mobile Radio Commun., Cannes, France, pp. 1-5.

[7] Jeyadeepan Jeganathan, Ali Ghrayeb, Leszek Szczecinski and Andres Ceron (2009), "Space Shift Keying Modulation for MIMO Channels" IEEE Transactions On Wireless Communications, Vol. 8, No. 7.

[8] Mesleh .R, Haas.H, Ahn.C, and Yun .S (2006), "Spatial modulation-a new low complexity spectral efficiency enhancing technique," in Proc. First International Conf. Commun. Netw., Beijing, China pp. 1-5.

[9] Ming Jiang, Member and Lajos Hanzo (2010), "Unitary Linear Dispersion Code Design and Optimization for MIMO Communication Systems" IEEE Signal Processing Letters, Vol. 17, No. 5.

[10] R. Rajashekar, K. Hari, and L. Hanzo (2011), "Field-extension-code-based dispersion matrices for coherently detected space-time shift keying," in Proc. IEEE GLOBECOM, Dec. 2011, pp. 1-5

[11] Raed Y. Mesleh, Harald Haas (2008), Sinan Sinanovi'c, Chang Wook Ahn, and Sangboh Yun, "Spatial Modulation" IEEE Transactions On Vehicular Technology, Vol. 57, No. 4.

[12] M. Di Renzo and H. Haas(2010), "Performance comparison of different spatial modulation schemes in correlated fading channels," in Proc. IEEE International Conf. Commun., Cape Town, South Africa, , pp. 1-6.

[13] Shinya Sugiura Sheng Chen and Lajos Hanzo(2010), "Coherent and differential spacetime shift keying: A dispersion matrix approach," 
IEEE Transactions On Communications, Vol. 58, No. 11.

[14] Tarokh .V, Alamouti .S, and Poon .P (1998), "New detection schemes for transmit diversity with no channel estimation," in Proc. International Conf. Universal Personal Communication., vol. 2,, pp. 917-920.

[15] Tarokh .V, Seshadri .N, and Calderbank .A (1998), "Space-time codes for high data rate wireless communication: performance criterion and code construction," IEEE Trans. Inf. Theory, vol. 44, no. 2, pp. 744-765.
[16] Tuchler .M (2004), "Design of serially concatenated systems depending on the block length," IEEE Trans. Commun., vol. 52, no. 2, pp. 209-218.

[17] Wilzeck .A, Cai .Q, Schiewer, .M and Kaiser .T (2007), "Effect of multiple-carrier frequency offsets in MIMO SC-FDMA systems," in Proc. Int. ITG/IEEE Workshop Smart Antennas, Vienna, Austria.

[18] Wolniansky .P, Foschini .G, Golden .G, and Valenzuela .R (1998), "V-BLAST: an architecture for realizing very high data rates over the richscattering wireless channel," in Proc. International Symp. Signals, Syst., Electron., Pisa, Italy, pp. 295300. 